\title{
Sex-specific responses of Populus deltoides to combined salinity and calcium under waterlogging conditions
}

\author{
L.-F. MIAO, D.-D. LI, F. YANG*, and Z.-H. TAN* \\ College of Ecology and Environment, Hainan University; Center for Eco-Environmental Restoration \\ Engineering of Hainan Province; Key Laboratory of Agro-Forestry Environmental Processes and Ecological \\ Regulation of Hainan Province, Haikou, Hainan 570228, P.R. China
}

\begin{abstract}
In this study, we investigated the sex-specific ion uptake and physiological and biochemical responses to combined salinity and calcium treatments of male and female Populus deltoides under waterlogging conditions. Results indicate that both $P$. deltoides clones were sensitive to salinity and calcium. Under well-watered conditions, salinity stress could especially increase the $\mathrm{Na}$ content in female $P$. deltoides clones, whereas salinity and calcium could increase the $\mathrm{K}$ and $\mathrm{Ca}$ content in male clones. Waterlogging could evidently stimulate Na content in leaves under salinity, especially in female $P$. deltoides clones. However, waterlogging had no visible effects on the amount of $\mathrm{Ca}$ absorption between the two clones under abundant calcium conditions. The physiological and biochemical responses to combined salinity and calcium exceeded the effects of salinity or calcium individually. However, no significant difference was observed, hence indicating the similar responses of male and female $P$. deltoides clones. The lower $\mathrm{Na}$ content and $\mathrm{Na}$ :Ca ratio, and the higher $\mathrm{K}$ and $\mathrm{Ca}$ content in male clones suggest that the male clones could maintain ion homeostasis better than the female clones. The sexspecific differences in net photosynthetic rate, intercellular $\mathrm{CO}_{2}$ concentration, effective quantum yield of photosystem II, photochemical quenching coefficient, non-photochemical quenching coefficient, photosynthetic electron transport rate, content of superoxide anions, hydroxyl radicals, $\mathrm{H}_{2} \mathrm{O}_{2}$, soluble protein, and activities of superoxide dismutase under certain conditions suggest that female $P$. deltoides clones are more sensitive to salinity, calcium, and the combination of both than their male counterparts.
\end{abstract}

Additional key words: combined abiotic stress, ion uptake, physiological responses, poplars, sexual dimorphisms.

\section{Introduction}

Populus deltoides is widely used as a desirable tree species for the construction of riparian-protective forests in Europe, North America, and China due to its fast growth, strong post-flooding recovery, and strong tolerance to waterlogging stress and winter flood (Gladwin and Roelle 1998, Cao and Conner 1999, Rowland 2001, Yang et al. 2011, Miao et al. 2017). However, waterlogging and salinity stresses often simultaneously occur in estuary and intertidal zones. Thus, whether $P$. deltoides could be used in the construction of riparian-protective forests in these specific regions remains unknown. The morphological, physiological, and biochemical responses to summer waterlogging, summer flooding, winter flooding, spring waterlogging, and post-flooding recovery and the ability of $P$. deltoides to adapt to these types of stresses have been well elucidated (Regehr et al. 1975, Cao and Conner 1999, Cooper et al. 2003, Yang et al. 2011, Yang et al. 2014, Miao et al. 2017). However, the mechanisms by which $P$. deltoides adapts to combined waterlogging and salinity stress are still unknown.

Calcium functions as an essential nutrient and a secondary messenger; it maintains the integrity and structure of membranes and cell walls, regulates plant growth and development, and mediates complex responses toward various developmental and environmental cues (Rengel 1992, Cramer 2002, Srivastava et al. 2013). Studies on exogenous calcium alleviating salinity stress in plants have been well documented (Rengel 1992, Lopez

Submitted 27 May 2020, last revision 22 July 2020, accepted 7 August 2020.

Abbreviations: APX - ascorbate peroxidase; $\mathrm{c}_{\mathrm{i}}$ - intercellular $\mathrm{CO}_{2}$ concentration; $\mathrm{E}$ - transpiration rate; ETR - photosynthetic electron transport rate; $\mathrm{F}_{0}$ - minimum fluorescence; $\mathrm{F}_{\mathrm{v}} / \mathrm{F}_{\mathrm{m}}$ - variable to maximum fluorescence ratio corresponding to maximum efficiency of photosystem II; $\mathrm{g}_{\mathrm{s}}$ - stomatal conductance; MDA - malondialdehyde; $\mathrm{O}_{2}{ }^{-}{ }^{-}$- superoxide anion radical; ${ }^{\circ} \mathrm{OH}$ - hydroxyl radical; $\mathrm{P}_{\mathrm{N}}-$ net photosynthetic rate; POD - peroxidase; PS - photosystem; qN - non-photochemical quenching; qP - photochemical quenching; SOD superoxide dismutase; WUEi- instantaneous water use efficiency; Yield - effective quantum yield of photosystem II.

Acknowledgements: This work was sponsored by the National Natural Science Foundation of China (No. 31660165, 32060240, and 31270449), and the Scientific Research Starting Foundation and Young Teachers Foundation of Hainan University to F. Yang (kyqd1573).

* Corresponding authors; e-mails: yangfan@hainanu.edu.cn; tanzh@xtbg.ac.cn 
and Satti 1996, Singh et al. 1999, Cramer 2002, Cabañero and Carvajal 2004, Murillo-Amador et al. 2006, Tuna et al. 2007, Tattini and Traversi 2009, Srivastava et al. 2013, An et al. 2014, Salahshoor and Kazemi 2016). Cramer (2002) described in detail the sodium-calcium interactions under salinity stress and suggested that these interactions could affect membrane properties, water and ion transport, nutrition, photosynthesis, and plant growth. However, different species, cultivars, and plant genotypes respond differently to supplemental Ca under salinity stress. Most species and genotypes respond positively to Ca application, whereas others respond negatively. In addition, $\mathrm{Ca}$ content and optimal $\mathrm{Na}$ :Ca ratio are important factors that improve salinity tolerance in plants; Ca content is usually in the range of $5-10 \mathrm{mM}$ and $\mathrm{Na}$ :Ca ratio $10-20$, depending on the salinity levels and genotypes (Cramer 2002, Srivastava et al. 2013). Therefore, whether $P$. deltoides is tolerant or sensitive to calcium and how it responds to exogenous calcium under saline conditions must be investigated.

Calcium alleviates damages brought about by waterlogging or flooding stresses in sweet potato (Lin et al. 2008), muskmelon (Gao et al. 2011), cucumber (He et al. 2012), soybean (Oh et al. 2014), and pepper (Yang et al. 2016). Oh et al. (2014) suggested that Ca might affect cell wall and hormone metabolisms, protein degradation/synthesis, and DNA synthesis in soybean roots under flooding stress. Yang et al. (2016) observed that exogenous $\mathrm{Ca}$ could alleviate waterlogging-induced damages to pepper plants by regulating the osmolyte content, antioxidant system activity, root respiration, and metabolism. However, responses to combined $\mathrm{Ca}$ and waterlogging stress need further research, especially in woody plant species. In particular, whether this combination is an independent process or causes additive effects must be determined. Seawater contains abundant sodium and calcium. Thus, the calcium content in estuary and intertidal zones is higher than in the riparian zones of freshwater rivers or lakes. The forests in estuary and intertidal zones often simultaneously encounter waterlogging, salinity, and excess of calcium. Although $P$. deltoides has good plasticity to waterlogging stress, its response to combined salinity and calcium under waterlogging condition must be defined.

Populus spp. are dioecious species. Different sexes and ages of poplars might employ different strategies to cope with waterlogging or flooding stress (Letts et al. 2008, Nielsen et al. 2010, Rood et al. 2010, Yang et al. 2011, Miao et al. 2017). Yang et al. (2011) and Miao et al. (2017) demonstrated that male $P$. deltoides clones have better waterlogging/flooding tolerance than female clones. However, Rood et al. (2010) and Nielsen et al. (2010) suggested that female $P$. angustifolia is more flood tolerant and successful in low and flood-prone sites than its male counterpart. Letts et al. (2008) reported that the photosynthetic gas exchange, leaf reflectance, chlorophyll fluorescence, and photosynthetic wateruse efficiency of male and female $P$. angustifolia clones in riparian woodland showed no significant difference. Juvany and Munné-Bosch (2015) suggested that general conclusions about sex-related stress tolerance in plants are not plausible. Sexual dimorphism may be related to species-specific differences, specific life-history traits, and adaptability to natural habitats. Therefore, sexual dimorphism and the ongoing multifactorial environmental stress must be further understood. Although the sexdependent responses of poplars to waterlogging stress have been compared, limited research has been conducted regarding the responses to combined salinity and calcium under waterlogging conditions. Consequently, the physiological mechanisms underlying sex-related differences in response to combined salinity and calcium stress under waterlogging conditions have not been fully understood.

$P$. deltoides is widely used in the Huaihe River and Yangtze River basin in China due to its fast growth rate and strong ability to overcome waterlogging stress. Male and female $P$. deltoides clones were used in this study to investigate sexual dimorphism when the plants are exposed to salinity, calcium, and combined salinity and calcium treatments under waterlogging condition. We hypothesized that sex-specific responses of $P$. deltoides to salinity, calcium, and combined salinity and calcium under well-watered and waterlogging conditions occurred, and the males would maintain ion homeostasis better than the females did. To confirm this hypothesis, the variations in ion uptake, photosynthesis, chlorophyll fluorescence, reactive oxygen species content, and antioxidant enzyme activities were analyzed.

\section{Materials and methods}

Plants and experimental design: The male (Populus deltoides clone Juba) and female $(P$. deltoides clone Danhong) were full-sib clones resulting from a cross between $P$. deltoides 2KEN8 ( $\hat{O}$, imported from Texas, USA) and $P$. deltoides 55/65 ( $\circ$, imported from Carbondale, USA). The female and male cuttings of $P$. deltoides originated from the artificial forests in Qianjiang $\left(30^{\circ} 09^{\prime} \mathrm{N}, 121^{\circ} 31^{\prime} \mathrm{E}\right)$, Hubei Province, China. The cuttings were planted into $10-\mathrm{dm}^{3}$ plastic pots filled with $10 \mathrm{~kg}$ of homogenized soil (one cutting per pot) on 1 March 2015. After sprouting and growing for 2 months, $P$. deltoides seedlings with similar crown sizes and equal heights (about $60 \mathrm{~cm}$ ) were selected for the experiments and placed in a natural environment with $1261 \mathrm{~mm}$ mean annual rainfall, $1494 \mathrm{~mm}$ annual evaporation, $80 \%$ annual relative humidity, and $16.9^{\circ} \mathrm{C}$ annual temperature.

The experimental layout was a randomized complete block design considering five main factors (sexual clones, watering regime, salinity, calcium, and combined salinity and calcium treatments). In the well-watered treatments, all pots were watered with fresh water or solutions every day, and excess water or solutions were allowed to drain through drainage holes into the dishes placed under the buckets. The excess water or solutions in the dishes were then re-used in the next watering to avoid loss of soil nutrients. For the salinity, calcium, and combined salinity and calcium treatments, $100 \mathrm{mM} \mathrm{NaCl}$ solutions, $10 \mathrm{mM}$ $\mathrm{CaCl}_{2}$ solutions, and mixture of both solutions were used 
for watering every other day, respectively. Fresh water was used for the other days. In the waterlogging treatments, the pots were watered with freshwater every day to $5 \mathrm{~cm}$ above the top soil. For the salinity, calcium, and combined salinity and calcium treatments, $\mathrm{NaCl}$ and/or $\mathrm{CaCl}_{2}$ were added in accordance with the water content at the initial stage of waterlogging treatment. The final concentrations of $\mathrm{NaCl}$ and $\mathrm{CaCl}_{2}$ were 100 and $10 \mathrm{mM}$, respectively. The pots subjected to salinity, calcium, and combined salinity and calcium treatments were watered with freshwater every day up to $5 \mathrm{~cm}$ above the soil during waterlogging. The plants were treated from 1 May to 15 May 2015. At the end of the 15-d treatment, gas exchange rate and chlorophyll fluorescence were measured, and fresh leaves were collected for analyses. Six replications with four cuttings each were used for each treatment.

Determination of $\mathrm{Na}, \mathrm{K}$, and $\mathrm{Ca}$ content in leaves: Sodium, potassium, and calcium were determined in accordance with the method of Williams and Twin (1960). The seventh mature leaves from the top of the plants were sampled. Powdered dry samples $(0.2 \mathrm{~g})$ were transferred into a dry and clean $100-\mathrm{cm}^{3}$ digestion vessel. A total of $10 \mathrm{~cm}^{3}$ of di-acid $\left(\mathrm{HNO}_{3}: \mathrm{HClO}_{4}\right.$ at the ratio of $\left.2: 1\right)$ mixture was added to the flask. The flasks were then heated at a temperature slowly raised to $200{ }^{\circ} \mathrm{C}$. Heating was stopped when dense white fumes of $\mathrm{HClO}_{4}$ were observed. The content of the flasks was boiled until it became clean and colorless. After cooling, the content was placed in a $50-\mathrm{cm}^{3}$ volumetric flask, which was filled up with deionized water to the marked spot. $\mathrm{Na}, \mathrm{K}$, and $\mathrm{Ca}$ content was measured from the digested leaf samples by using a flame photometer (M410, Sherwood, OR, USA). The concentrations were calculated using standard curves.

Gas exchange measurements: The fourth fully expanded leaves from six cuttings of each treatment were selected for measuring gas exchange parameters. Net photosynthetic rate $\left(\mathrm{P}_{\mathrm{N}}\right)$, stomatal conductance $\left(\mathrm{g}_{\mathrm{s}}\right)$, intercellular $\mathrm{CO}_{2}$ concentration $\left(\mathrm{c}_{\mathrm{i}}\right)$, and transpiration $(\mathrm{E})$ were measured from 9:00 to 11:30 using a LI-COR 6400 portable photosynthesis system (LI-COR, Lincoln, NE, USA). The photosynthetically active radiation was set to $1400 \mu \mathrm{mol} \mathrm{m} \mathrm{m}^{-2} \cdot \mathrm{s}^{-1}$, provided by a $6400-02 \mathrm{LED}$ radiation source. The flow rate of air through the sample chamber was set at $500 \mu \mathrm{mol} \mathrm{m} \mathrm{m}^{-2} \mathrm{~s}^{-1}$, and the leaf temperature and relative humidity were maintained at $25 \pm 0.8{ }^{\circ} \mathrm{C}$ and $50 \%$, respectively (Yang et al. 2011). Instantaneous water use efficiency $\left(\mathrm{WUEi}=\mathrm{P}_{\mathrm{N}} / \mathrm{E}\right)$ was calculated.

Chlorophyll fluorescence measurements: The same leaves used for gas exchange were selected for the measurements of chlorophyll fluorescence parameters: maximum efficiency of photosystem (PS) II (variable to maximum fluorescence ratio $\mathrm{F}_{\mathrm{v}} / \mathrm{F}_{\mathrm{m}}$ ), effective quantum yield of PS II (Yield), non-photochemical quenching coefficient (qN), photochemical quenching coefficient (qP), and photosynthetic electron transport rate (ETR) using a pulse amplitude modulation chlorophyll fluorometer (PAM 2100, Walz, Effeltrich, Germany). The leaf samples were placed in darkness by covering them with aluminum foil for $30 \mathrm{~min}$, followed by measurement of minimum fluorescence $\left(\mathrm{F}_{0}\right)$ at $250 \mu \mathrm{mol} \mathrm{m} \mathrm{m}^{-2} \mathrm{~s}^{-1}$ of photosynthetic photon flux density (PPFD) and maximum fluorescence $\left(\mathrm{F}_{\mathrm{m}}\right)$ following a saturating pulse of actinic light of $2400 \mu \mathrm{mol} \mathrm{m} \mathrm{m}^{-2} \mathrm{~s}^{-1}$ PPFD (Yang et al. 2011). The measurements were carried out between 8:00 and 11:00.

Determination of reactive oxygen species (ROS) and malondialdehyde (MDA) content: Detections of hydrogen peroxide $\left(\mathrm{H}_{2} \mathrm{O}_{2}\right)$, superoxide radical anions $\left(\mathrm{O}_{2}{ }^{-{ }^{-}}\right)$, hydroxyl radicals $(\mathrm{OH})$, and MDA content were based on spectrometric procedures described previously (Yang et al. 2011, 2015, Han et al. 2015).

Determination of soluble protein content and antioxidant enzyme activities: Approximately $1 \mathrm{~g}$ of fresh samples were ground with liquid nitrogen and then homogenized in $10 \mathrm{~cm}^{3}$ of $100 \mathrm{mM}$ universal sodium phosphate extraction buffer as described by Han et al. (2015) and Yang et al (2015). The supernatant $\left(0.5 \mathrm{~cm}^{3}\right)$ was stored at $-80^{\circ} \mathrm{C}$ until it was used for the determination of soluble protein and ROS content, and antioxidant enzyme activities. The soluble protein was quantified using the Bradford method (Bradford 1976). The antioxidant enzyme activities, including peroxidase (POD), superoxide dismutase (SOD), and ascorbate peroxidase (APX), were determined spectrophotometrically in accordance with the manufacturer's instructions and as described by Yang et al. (2011, 2015) and Han et al. (2015). One unit (U) of SOD activity was defined as the amount of the enzyme inhibiting photoreduction of nitroblue tetrazolium by $50 \%$. One unit of POD activity was defined as the amount of the enzyme causing a change in absorbance of 0.01 at $470 \mathrm{~nm}$. One unit of APX was defined as the amount of the enzyme causing a change in absorbance of 0.01 at $290 \mathrm{~nm}$.

Statistical analyses: The results were expressed as means \pm standard errors $(n=6)$. The SPSS 16.0 software was used for statistical analysis. Post hoc multiple comparison from the general linear model was used to evaluate the effects of sex, waterlogging, salt, calcium, and their interaction, and the Tukey's test was employed to test the sex and treatment differences. Differences were considered significant at $P<0.05$.

\section{Results}

Significant visible damages, such as leaf chlorosis, leaf necrosis, or leaf abscission, were observed when plants were exposed to salinity, calcium, and combined salinity and calcium whether under well-watered or waterlogging conditions. However, all plants could survive well after being exposed only to waterlogging. No significant morphological variations were observed among the plants under all treatments. However, insignificant differences in morphological traits were found between female and male clones under the same treatment.

Under well-watered conditions, salinity and combined 
Table 1. The variations of $\mathrm{Na}, \mathrm{K}$, and $\mathrm{Ca}$ content, and $\mathrm{Na}$ :Ca ratio in leaves of male and female Populus deltoides. Means $\pm \mathrm{SEs}, n=6$; different letters indicate significant differences at $P<0.05$ according to Tukey's test; * $-P \leq 0.05, * *-P \leq 0.01, * * *-\mathrm{P} \leq 0.001$, ns - not significant difference. $\mathrm{F}_{\mathrm{S}}$ - sex effect, $\mathrm{F}_{\mathrm{W}}$ - waterlogging effect, $\mathrm{F}_{\mathrm{Na}}$ - salt effect, $\mathrm{F}_{\mathrm{Ca}}$ - calcium effect, $\mathrm{F}_{\mathrm{Na} \times \mathrm{Ca}}$ - salt $\times$ calcium interaction, $\mathrm{F}_{\mathrm{S} \times \mathrm{W}}-\operatorname{sex} \times$ waterlogging interaction, $\mathrm{F}_{\mathrm{S} \times \mathrm{Na}}-\operatorname{sex} \times$ salt interaction, $\mathrm{F}_{\mathrm{S} \times \mathrm{Ca}}-\operatorname{sex} \times$ calcium interaction, $\mathrm{F}_{\mathrm{S} \times \mathrm{Na} \times \mathrm{Ca}}-\operatorname{sex} \times$ salt $\times$ calcium interaction, $\mathrm{F}_{\mathrm{W} \times \mathrm{Na}}$ - waterlogging $\times$ salt interaction, $\mathrm{F}_{\mathrm{W} \times \mathrm{Ca}}$ - waterlogging $\times$ calcium interaction, $\mathrm{F}_{\mathrm{W} \times \mathrm{Na} \times \mathrm{Ca}}$ - waterlogging $\times$ salt $\times$ calcium interaction, $\mathrm{F}_{\mathrm{S} \times \mathrm{W} \times \mathrm{Na} \times \mathrm{Ca}}-\mathrm{sex} \times$ waterlogging $\times$ salt $\times$ calcium interaction.

\begin{tabular}{|c|c|c|c|c|c|c|}
\hline Water regime & Treatment & Sex & $\mathrm{Na}\left[\mathrm{mg} \mathrm{g}^{-1}\right.$ (d.m.) $]$ & $\mathrm{K}\left[\mathrm{mg} \mathrm{g}^{-1}\right.$ (d.m.) $]$ & $\mathrm{Ca}\left[\mathrm{mg} \mathrm{g}^{-1}\right.$ (d.m.) $]$ & $\mathrm{Na}: \mathrm{Ca}$ ratio \\
\hline \multirow[t]{8}{*}{ Well-watered } & \multirow[t]{2}{*}{ control } & female & $0.71 \pm 0.02 \mathrm{~g}$ & $9.93 \pm 0.11 \mathrm{~d}$ & $10.33 \pm 0.09 \mathrm{i}$ & $0.07 \pm 0.0016 \mathrm{fgh}$ \\
\hline & & male & $0.45 \pm 0.01 \mathrm{~g}$ & $10.47 \pm 0.13 \mathrm{~d}$ & $10.88 \pm 0.14 \mathrm{i}$ & $0.04 \pm 0.0007 \mathrm{gh}$ \\
\hline & \multirow[t]{2}{*}{$\mathrm{NaCl}$} & female & $2.18 \pm 0.08 \mathrm{e}$ & $8.73 \pm 0.12$ ef & $12.56 \pm 0.11 \mathrm{f}$ & $0.12 \pm 0.0017 \mathrm{def}$ \\
\hline & & male & $1.48 \pm 0.01 \mathrm{f}$ & $13.03 \pm 0.31 \mathrm{a}$ & $14.15 \pm 0.27 \mathrm{~d}$ & $0.15 \pm 0.0051 \mathrm{de}$ \\
\hline & \multirow[t]{2}{*}{$\mathrm{Ca}$} & female & $0.60 \pm 0.02 \mathrm{~g}$ & $10.46 \pm 0.22 \mathrm{~d}$ & $12.08 \pm 0.21 \mathrm{fgh}$ & $0.05 \pm 0.0011 \mathrm{gh}$ \\
\hline & & male & $0.86 \pm 0.06 \mathrm{~g}$ & $12.79 \pm 0.26 \mathrm{ab}$ & $12.40 \pm 0.41 \mathrm{fg}$ & $0.07 \pm 0.0045 \mathrm{fgh}$ \\
\hline & \multirow[t]{2}{*}{$\mathrm{NaCl}+\mathrm{Ca}$} & female & $2.01 \pm 0.03 \mathrm{e}$ & $11.92 \pm 0.06 \mathrm{c}$ & $11.87 \pm 0.23 \mathrm{fgh}$ & $0.17 \pm 0.001 \mathrm{~d}$ \\
\hline & & male & $1.50 \pm 0.02 \mathrm{f}$ & $12.33 \pm 0.21 \mathrm{bc}$ & $14.19 \pm 0.25 \mathrm{~d}$ & $0.11 \pm 0.0026 \mathrm{efg}$ \\
\hline \multirow[t]{21}{*}{ Waterlogging } & \multirow[t]{2}{*}{ control } & female & $0.67 \pm 0.02 \mathrm{~g}$ & $12.88 \pm 0.28 \mathrm{ab}$ & $20.38 \pm 0.23 \mathrm{~b}$ & $0.03 \pm 0.0007 \mathrm{~h}$ \\
\hline & & male & $0.61 \pm 0.02 \mathrm{~g}$ & $13.16 \pm 0.11 \mathrm{a}$ & $21.29 \pm 0.21 \mathrm{a}$ & $0.03 \pm 0.001 \mathrm{~h}$ \\
\hline & \multirow[t]{2}{*}{$\mathrm{NaCl}$} & female & $26.51 \pm 0.29 \mathrm{~b}$ & $6.60 \pm 0.14 \mathrm{j}$ & $13.42 \pm 0.20 \mathrm{e}$ & $1.98 \pm 0.0162 \mathrm{~b}$ \\
\hline & & male & $25.44 \pm 0.46 \mathrm{c}$ & $9.25 \pm 0.23 \mathrm{e}$ & $13.58 \pm 0.25 \mathrm{de}$ & $1.87 \pm 0.0661 \mathrm{c}$ \\
\hline & \multirow[t]{2}{*}{$\mathrm{Ca}$} & female & $0.90 \pm 0.01 \mathrm{~g}$ & $7.69 \pm 0.16 \mathrm{hi}$ & $11.68 \pm 0.15 \mathrm{~h}$ & $0.08 \pm 0.0003 \mathrm{fgh}$ \\
\hline & & male & $0.95 \pm 0.07 \mathrm{~g}$ & $7.39 \pm 0.16 \mathrm{i}$ & $12.13 \pm 0.08 \mathrm{fgh}$ & $0.08 \pm 0.0055 \mathrm{fgh}$ \\
\hline & \multirow[t]{15}{*}{$\mathrm{NaCl}+\mathrm{Ca}$} & female & $31.84 \pm 0.23 \mathrm{a}$ & $8.51 \pm 0.10 \mathrm{fg}$ & $11.78 \pm 0.13 \mathrm{gh}$ & $2.08 \pm 0.0056 \mathrm{a}$ \\
\hline & & male & $24.54 \pm 0.31 \mathrm{~d}$ & $8.16 \pm 0.12 \mathrm{gh}$ & $16.88 \pm 0.25 \mathrm{c}$ & $1.89 \pm 0.0397 \mathrm{c}$ \\
\hline & & $P>\mathrm{F}_{\mathrm{S}}$ & $0.000 * * *$ & $0.000 * * *$ & $0.177 \mathrm{~ns}$ & $0.487 \mathrm{~ns}$ \\
\hline & & $P>\mathrm{F}_{\mathrm{W}}$ & $0.000 * * *$ & $0.000 * * *$ & $0.000 * * *$ & $0.000 * * *$ \\
\hline & & $P>\mathrm{F}_{\mathrm{Na}}$ & $0.000 * * *$ & $0.000 * * *$ & $0.003 * *$ & $0.000 * * *$ \\
\hline & & $P>\mathrm{F}_{\mathrm{Ca}}$ & $0.000 * * *$ & $0.000 * * *$ & $0.000 * * *$ & $0.008^{* *}$ \\
\hline & & $P>\mathrm{F}_{\mathrm{Na} \times \mathrm{Ca}}$ & $0.000 * * *$ & $0.000 * * *$ & $0.000 * * *$ & $0.788 \mathrm{~ns}$ \\
\hline & & $P>\mathrm{F}_{\mathrm{S} \times \mathrm{W}}$ & $0.000 * * *$ & $0.000 * * *$ & $0.000 * * *$ & $0.120 \mathrm{~ns}$ \\
\hline & & $P>\mathrm{F}_{\mathrm{S} \times \mathrm{Na}}$ & $0.000 * * *$ & $0.000 * * *$ & $0.001 * * *$ & $0.325 \mathrm{~ns}$ \\
\hline & & $P>F_{\mathrm{S} \times \mathrm{Ca}}$ & $0.000 * * *$ & $0.000 * * *$ & $0.000 * * *$ & $0.004 * * *$ \\
\hline & & $P>F_{\mathrm{S} \times \mathrm{Na} \times \mathrm{Ca}}$ & $0.000 * * *$ & $0.000 * * *$ & $0.000 * * *$ & $0.078 \mathrm{~ns}$ \\
\hline & & $P>F_{\mathrm{W} \times \mathrm{Na}}$ & $0.000 * * *$ & $0.000 * * *$ & $0.000 * * *$ & $0.000 * * *$ \\
\hline & & $P>F_{\mathrm{W} \times \mathrm{Ca}}$ & $0.000 * * *$ & $0.000 * * *$ & $0.000 * * *$ & $0.016^{*}$ \\
\hline & & $P>F_{\mathrm{W} \times \mathrm{Na} \times \mathrm{Ca}}$ & $0.000 * * *$ & $0.000 * * *$ & $0.000 * * *$ & $0.689 \mathrm{~ns}$ \\
\hline & & $P>\mathrm{F}_{\mathrm{S} \times \mathrm{W} \times \mathrm{Na} \times \mathrm{Ca}}$ & $0.000 * * *$ & $0.000 * * *$ & $0.000 * * *$ & $0.000 * * *$ \\
\hline
\end{tabular}

salinity and calcium treatments significantly increased the Na content to approximately threefold in female and male P. deltoides clones compared with their controls (Table 1), whereas calcium treatment exhibited no significant effect on the $\mathrm{Na}$ content of both clones. Thus, the $\mathrm{Na}$ content in salinity and combined salinity and $\mathrm{Ca}$ treatments was significantly higher than that in only $\mathrm{Ca}$ treatment for both clones. In addition, the female clones had significantly higher $\mathrm{Na}$ content than male clones after salinity and combined salinity and $\mathrm{Ca}$ treatments.

Salinity and combined salinity and $\mathrm{Ca}$ treatments under waterlogging conditions could substantially stimulate $\mathrm{Na}$ uptake in female and male clones. Both treatments increased the Na content to more than 12-fold in both clones compared with their individual controls under well-watered conditions. However, waterlogging and $\mathrm{Ca}$ treatment under waterlogging caused no effect on the $\mathrm{Na}$ content of both clones compared with their individual controls. In addition, the combined salinity and $\mathrm{Ca}$ treatment could significantly stimulate $\mathrm{Na}$ uptake in female clones but significantly inhibit the same process in male clones compared with salinity treatment under waterlogging. The female clones had significantly higher Na content than males after salinity and combined salinity and $\mathrm{Ca}$ treatments under waterlogging.

Under well-watered conditions, the salinity, Ca, and combined salinity and $\mathrm{Ca}$ treatments significantly increased the $\mathrm{K}$ content (Table 1) of male clones compared with the controls. Meanwhile, the $\mathrm{K}$ content in female clones was significantly increased by the combined salinity and $\mathrm{Ca}$ treatment, but significantly inhibited by salinity treatment, and not significantly affected by $\mathrm{Ca}$ treatment. No significant difference in $\mathrm{K}$ content was observed among male clones in different treatments. In addition, the male clones had significantly higher $\mathrm{K}$ content than the female clones after salinity and $\mathrm{Ca}$ treatments. 
Table 2. Net photosynthetic rate $\left(\mathrm{P}_{\mathrm{N}}\right)$, stomatal conductance $\left(\mathrm{g}_{\mathrm{s}}\right)$, intrinsic water use efficiency (WUEi), transpiration rate (E), and intercellular $\mathrm{CO}_{2}$ concentration $\left(\mathrm{c}_{\mathrm{i}}\right)$ variations in male and female Populus deltoides. Means $\pm \mathrm{SEs}, n=6$; different letters indicate significant differences at $P<0.05$ according to Tukey's test. For explanation of abbreviations - see Table 1 .

\begin{tabular}{|c|c|c|c|c|c|c|c|}
\hline \multirow{2}{*}{$\frac{\text { Water regime }}{\text { Well-watered }}$} & \multirow{2}{*}{$\begin{array}{l}\text { Treatment } \\
\text { control }\end{array}$} & \multirow{2}{*}{$\begin{array}{l}\text { Sex } \\
\text { female }\end{array}$} & \multirow{2}{*}{$\frac{\mathrm{P}_{\mathrm{N}}\left[\mu \mathrm{mol} \mathrm{m} \mathrm{m}^{-2} \mathrm{~s}^{-1}\right]}{18.43 \pm 1.28 \mathrm{a}}$} & \multirow{2}{*}{$\frac{\mathrm{g}_{\mathrm{s}}\left[\mathrm{mol} \mathrm{m}^{-2} \mathrm{~s}^{-1}\right]}{0.59 \pm 0.11 \mathrm{a}}$} & \multirow{2}{*}{$\frac{\text { WUEi }\left[\mu \mathrm{mol} \mathrm{mmol}{ }^{-1}\right]}{4.56 \pm 0.78 \mathrm{a}}$} & \multicolumn{2}{|c|}{$\mathrm{E}\left[\mathrm{mmol} \mathrm{m} \mathrm{s}^{-1}\right] \mathrm{c}_{\mathrm{i}}\left[\mu \mathrm{mol} \mathrm{mol} \mathrm{l}^{-1}\right]$} \\
\hline & & & & & & $4.33 \pm 0.92 \mathrm{de}$ & $300.55 \pm 11.94 \mathrm{ab}$ \\
\hline & & male & $16.30 \pm 0.26 \mathrm{ab}$ & $0.47 \pm 0.04 \mathrm{ab}$ & $3.54 \pm 0.46 \mathrm{bc}$ & $4.73 \pm 0.49$ cde & $315.52 \pm 7.65 \mathrm{ab}$ \\
\hline & $\mathrm{NaCl}$ & female & $11.99 \pm 0.89 \mathrm{de}$ & $0.63 \pm 0.04 \mathrm{a}$ & $1.98 \pm 0.12 \mathrm{e}$ & $8.10 \pm 0.26 \mathrm{a}$ & $310.32 \pm 6.08 \mathrm{ab}$ \\
\hline & & male & $16.01 \pm 1.14 \mathrm{ab}$ & $0.49 \pm 0.05 \mathrm{ab}$ & $1.63 \pm 0.03 \mathrm{e}$ & $7.41 \pm 0.67 \mathrm{ab}$ & $315.80 \pm 3.79 \mathrm{ab}$ \\
\hline & $\mathrm{Ca}$ & female & $14.28 \pm 1.23 \mathrm{bcd}$ & $0.58 \pm 0.04 \mathrm{a}$ & $1.88 \pm 0.13 \mathrm{e}$ & $7.61 \pm 0.44 \mathrm{ab}$ & $312.84 \pm 6.56 \mathrm{ab}$ \\
\hline & & male & $13.97 \pm 0.16 \mathrm{bcd}$ & $0.57 \pm 0.06 \mathrm{a}$ & $1.70 \pm 0.07 \mathrm{e}$ & $8.26 \pm 0.41 \mathrm{a}$ & $320.20 \pm 4.56 \mathrm{a}$ \\
\hline & $\mathrm{NaCl}+\mathrm{Ca}$ & female & $15.44 \pm 1.53 \mathrm{abc}$ & $0.50 \pm 0.08 \mathrm{ab}$ & $2.13 \pm 0.15 \mathrm{e}$ & $7.28 \pm 0.76 \mathrm{ab}$ & $299.64 \pm 3.71 \mathrm{ab}$ \\
\hline & & male & $14.68 \pm 0.86 \mathrm{bcd}$ & $0.52 \pm 0.05 \mathrm{ab}$ & $1.87 \pm 0.18 \mathrm{e}$ & $7.89 \pm 0.29 \mathrm{ab}$ & $312.30 \pm 9.45 \mathrm{ab}$ \\
\hline \multirow[t]{21}{*}{ Waterlogging } & control & female & $13.30 \pm 0.89 \mathrm{bcd}$ & $0.47 \pm 0.06 \mathrm{ab}$ & $3.74 \pm 0.13 \mathrm{ab}$ & $3.58 \pm 0.35 \mathrm{e}$ & $291.19 \pm 5.31 \mathrm{ab}$ \\
\hline & & male & $14.46 \pm 0.19 \mathrm{bcd}$ & $0.48 \pm 0.05 \mathrm{ab}$ & $3.41 \pm 0.36 \mathrm{bc}$ & $4.33 \pm 0.41 \mathrm{de}$ & $280.24 \pm 5.53 \mathrm{bc}$ \\
\hline & $\mathrm{NaCl}$ & female & $11.77 \pm 0.39 \mathrm{de}$ & $0.26 \pm 0.07 \mathrm{c}$ & $3.26 \pm 0.43 \mathrm{bcd}$ & $3.78 \pm 0.67 \mathrm{e}$ & $294.44 \pm 13.4 \mathrm{ab}$ \\
\hline & & male & $13.66 \pm 0.94 \mathrm{bcd}$ & $0.34 \pm 0.02 \mathrm{bc}$ & $2.64 \pm 0.13 \mathrm{cde}$ & $5.19 \pm 0.37 \mathrm{cde}$ & $296.04 \pm 6.75 \mathrm{ab}$ \\
\hline & $\mathrm{Ca}$ & female & $14.39 \pm 1.38 \mathrm{bcd}$ & $0.46 \pm 0.08 \mathrm{ab}$ & $2.26 \pm 0.26 \mathrm{de}$ & $6.48 \pm 0.71 \mathrm{abc}$ & $299.90 \pm 9.8 \mathrm{ab}$ \\
\hline & & male & $11.81 \pm 0.57 \mathrm{de}$ & $0.36 \pm 0.06 \mathrm{bc}$ & $1.97 \pm 0.11 \mathrm{e}$ & $6.05 \pm 0.58 \mathrm{bcd}$ & $303.10 \pm 8.72 \mathrm{ab}$ \\
\hline & $\mathrm{NaCl}+\mathrm{Ca}$ & female & $12.52 \pm 1.49 \mathrm{cde}$ & $0.24 \pm 0.08 \mathrm{c}$ & $3.33 \pm 0.55 \mathrm{bcd}$ & $3.99 \pm 0.84 \mathrm{e}$ & $254.44 \pm 23.51 \mathrm{c}$ \\
\hline & & male & $10.08 \pm 0.39 \mathrm{e}$ & $0.26 \pm 0.04 \mathrm{c}$ & $2.60 \pm 0.4$ cde & $4.03 \pm 0.51 \mathrm{e}$ & $291.93 \pm 17.2 \mathrm{ab}$ \\
\hline & & $P>\mathrm{F}_{\mathrm{S}}$ & $0.023^{*}$ & $0.314 \mathrm{~ns}$ & $0.008^{* *}$ & $0.243 \mathrm{~ns}$ & $0.093 \mathrm{~ns}$ \\
\hline & & $P>\mathrm{F}_{\mathrm{W}}$ & $0.000 * * *$ & $0.000 * * *$ & $0.006^{* *}$ & $0.000 * * *$ & $0.000 * * *$ \\
\hline & & $P>\mathrm{F}_{\mathrm{Na}}$ & $0.009^{* *}$ & $0.005 * *$ & $0.011^{*}$ & $0.327 \mathrm{~ns}$ & $0.25 \mathrm{~ns}$ \\
\hline & & $P>\mathrm{F}_{\mathrm{Ca}}$ & $0.03 *$ & $0.309 \mathrm{~ns}$ & $0.000 * * *$ & $0.000 * * *$ & $0.816 \mathrm{~ns}$ \\
\hline & & $P>\mathrm{F}_{\mathrm{Na} \times \mathrm{Ca}}$ & $0.066 \mathrm{~ns}$ & $0.556 \mathrm{~ns}$ & $0.000 * * *$ & $0.000 * * *$ & $0.015^{*}$ \\
\hline & & $P>\mathrm{F}_{\mathrm{S} \times \mathrm{W}}$ & $0.181 \mathrm{~ns}$ & $0.304 \mathrm{~ns}$ & $0.913 \mathrm{~ns}$ & $0.733 \mathrm{~ns}$ & $0.827 \mathrm{~ns}$ \\
\hline & & $P>\mathrm{F}_{\mathrm{S} \times \mathrm{Na}}$ & $0.711 \mathrm{~ns}$ & $0.422 \mathrm{~ns}$ & $0.912 \mathrm{~ns}$ & $0.998 \mathrm{~ns}$ & $0.312 \mathrm{~ns}$ \\
\hline & & $P>\mathrm{F}_{\mathrm{S} \times \mathrm{Ca}}$ & $0.444 \mathrm{~ns}$ & $0.712 \mathrm{~ns}$ & $0.518 \mathrm{~ns}$ & $0.666 \mathrm{~ns}$ & $0.241 \mathrm{~ns}$ \\
\hline & & $P>\mathrm{F}_{\mathrm{S} \times \mathrm{Na} \times \mathrm{Ca}}$ & $0.827 \mathrm{~ns}$ & $0.654 \mathrm{~ns}$ & $0.512 \mathrm{~ns}$ & $0.714 \mathrm{~ns}$ & $0.386 \mathrm{~ns}$ \\
\hline & & $P>\mathrm{F}_{\mathrm{W} \times \mathrm{Na}}$ & $0.78 \mathrm{~ns}$ & $0.024 *$ & $0.002 * *$ & $0.000 * * *$ & $0.527 \mathrm{~ns}$ \\
\hline & & $P>\mathrm{F}_{\mathrm{W} \times \mathrm{Ca}}$ & $0.995 \mathrm{~ns}$ & $0.411 \mathrm{~ns}$ & $0.365 \mathrm{~ns}$ & $0.232 \mathrm{~ns}$ & $0.714 \mathrm{~ns}$ \\
\hline & & $P>\mathrm{F}_{\mathrm{W} \times \mathrm{Na} \times \mathrm{Ca}}$ & $0.015^{*}$ & $0.404 \mathrm{~ns}$ & $0.149 \mathrm{~ns}$ & $0.497 \mathrm{~ns}$ & $0.291 \mathrm{~ns}$ \\
\hline & & $P>\mathrm{F}_{\mathrm{S} \times \mathrm{W} \times \mathrm{Na} \times \mathrm{Ca}}$ & $0.013^{*}$ & $0.288 \mathrm{~ns}$ & $0.631 \mathrm{~ns}$ & $0.235 \mathrm{~ns}$ & $0.403 \mathrm{~ns}$ \\
\hline
\end{tabular}

Waterlogging stimulated $\mathrm{K}$ uptake in female and male clones compared with the controls. However, the salinity, calcium, and combined salinity and Ca treatments under waterlogging could significantly inhibit $\mathrm{K}$ uptake in both clones. Several differences in $\mathrm{K}$ content were found in both clones after all three treatments under waterlogging. In addition, the male clones had a significantly higher $\mathrm{K}$ content than the female clone after salinity treatment under waterlogging.

Under well-watered conditions, the $\mathrm{Ca}$ content (Table 1) of female and male clones significantly increased in salinity, $\mathrm{Ca}$, and combined salinity and $\mathrm{Ca}$ treatments compared with the controls. No significant differences in $\mathrm{Ca}$ content were noted among female clones under different treatments. In addition, the male clones had higher Ca content than the female clones in each treatment, and significantly higher Ca content was detected in salinity and combined salinity and $\mathrm{Ca}$ treatments.

Waterlogging stimulated Ca uptake in female and male clones compared with the controls. However, the salinity, $\mathrm{Ca}$, and combined salinity and $\mathrm{Ca}$ treatments under waterlogging could not significantly affect $\mathrm{Ca}$ uptake in both clones compared with their individual controls under well-watered conditions. Differences in Ca content were observed among the treatments under waterlogging. In addition, the male clones had a significantly higher $\mathrm{Ca}$ content than the female clones in the combined salinity and $\mathrm{Ca}$ treatment under waterlogging, whereas no significant differences in $\mathrm{Ca}$ content were found between the clones in salinity and $\mathrm{Ca}$ treatments.

Under well-watered conditions, salinity and combined salinity and Ca treatments significantly increased the ratio of $\mathrm{Na}: \mathrm{Ca}$ (Table 1) in female and male clones compared with their controls, whereas $\mathrm{Ca}$ treatment exhibited no significant effect on the ratio of $\mathrm{Na}: \mathrm{Ca}$ in both clones. Thus, the values of the $\mathrm{Na}: \mathrm{Ca}$ in salinity and combined salinity and $\mathrm{Ca}$ treatments was significantly higher than in $\mathrm{Ca}$ treatment alone for both clones. In addition, the female clones had significantly higher values of $\mathrm{Na}$ : $\mathrm{Ca}$ than male clones in combined salinity and $\mathrm{Ca}$ treatments.

Salinity and combined salinity and $\mathrm{Ca}$ treatments under waterlogging substantially increased the ratio 
Table 3. Maximum efficiency of photosystem II $\left(\mathrm{F}_{\mathrm{v}} / \mathrm{F}_{\mathrm{m}}\right)$, effective quantum yield of PS II (Yield), photochemical quenching (qP), nonphotochemical quenching (qN), and photosynthetic electron transport rate (ETR) variations in male and female Populus deltoides. Values are means $\pm \operatorname{SE}(n=6)$; different letters indicate significant differences at $P<0.05$ according to Tukey's test. For explanation of abbreviations - see Table 1 .

\begin{tabular}{|c|c|c|c|c|c|c|c|}
\hline $\begin{array}{l}\text { Water } \\
\text { regime }\end{array}$ & Treatment & Sex & $\mathrm{F}_{\mathrm{v}} / \mathrm{F}_{\mathrm{m}}$ & Yield & $\mathrm{qP}$ & $\mathrm{qN}$ & ETR \\
\hline \multirow{8}{*}{$\begin{array}{l}\text { Well- } \\
\text { watered }\end{array}$} & \multirow[t]{2}{*}{ control } & female & $0.762 \pm 0.011 \mathrm{~b}$ & $0.618 \pm 0.028 \mathrm{abcd}$ & $0.899 \pm 0.023 \mathrm{abcd}$ & $0.280 \pm 0.043 \mathrm{efg}$ & $67.333 \pm 3.383 \mathrm{a}$ \\
\hline & & male & $0.778 \pm 0.002 \mathrm{ab}$ & $0.656 \pm 0.01 \mathrm{ab}$ & $0.928 \pm 0.007 \mathrm{a}$ & $0.231 \pm 0.031 \mathrm{efg}$ & $65.000 \pm 1.155 \mathrm{abc}$ \\
\hline & \multirow[t]{2}{*}{$\mathrm{NaCl}$} & female & $0.780 \pm 0.005 \mathrm{ab}$ & $0.613 \pm 0.024 \mathrm{abcd}$ & $0.902 \pm 0.012 \mathrm{abc}$ & $0.378 \pm 0.061 \mathrm{cde}$ & $60.667 \pm 2.603 \mathrm{abcd}$ \\
\hline & & male & $0.775 \pm 0.008 \mathrm{ab}$ & $0.528 \pm 0.019 \mathrm{fg}$ & $0.865 \pm 0.011 \mathrm{defg}$ & $0.574 \pm 0.024 \mathrm{ab}$ & $52.333 \pm 1.764$ ef \\
\hline & \multirow[t]{2}{*}{$\mathrm{Ca}$} & female & $0.778 \pm 0.007 \mathrm{ab}$ & $0.622 \pm 0.011 \mathrm{abcd}$ & $0.906 \pm 0.009 \mathrm{abc}$ & $0.334 \pm 0.017 \mathrm{def}$ & $61.667 \pm 1.333 \mathrm{abcd}$ \\
\hline & & male & $0.779 \pm 0.007 \mathrm{ab}$ & $0.648 \pm 0.008 \mathrm{ab}$ & $0.920 \pm 0.009 \mathrm{ab}$ & $0.305 \pm 0.036 \mathrm{defg}$ & $64.333 \pm 0.882 \mathrm{abc}$ \\
\hline & \multirow[t]{2}{*}{$\mathrm{NaCl}+\mathrm{Ca}$} & female & $0.788 \pm 0.005 \mathrm{ab}$ & $0.639 \pm 0.005 \mathrm{abc}$ & $0.908 \pm 0.004 \mathrm{abc}$ & $0.311 \pm 0.012 \mathrm{defg}$ & $63.333 \pm 0.333 \mathrm{abc}$ \\
\hline & & male & $0.788 \pm 0.005 a b$ & $0.647 \pm 0.021 \mathrm{ab}$ & $0.914 \pm 0.01 \mathrm{abc}$ & $0.293 \pm 0.039 \mathrm{efg}$ & $64.333 \pm 2.186 \mathrm{abc}$ \\
\hline \multirow{21}{*}{$\begin{array}{l}\text { Water- } \\
\text { logging }\end{array}$} & \multirow[t]{2}{*}{ control } & female & $0.778 \pm 0.007 \mathrm{ab}$ & $0.653 \pm 0.011 \mathrm{ab}$ & $0.920 \pm 0.005 \mathrm{ab}$ & $0.196 \pm 0.034 \mathrm{fg}$ & $65.000 \pm 1.155 \mathrm{abc}$ \\
\hline & & male & $0.783 \pm 0.004 \mathrm{ab}$ & $0.663 \pm 0.017 \mathrm{a}$ & $0.926 \pm 0.008 \mathrm{a}$ & $0.183 \pm 0.071 \mathrm{~g}$ & $65.667 \pm 1.856 \mathrm{ab}$ \\
\hline & \multirow[t]{2}{*}{$\mathrm{NaCl}$} & female & $0.776 \pm 0.007 \mathrm{ab}$ & $0.505 \pm 0.037 \mathrm{~g}$ & $0.848 \pm 0.022 \mathrm{~g}$ & $0.551 \pm 0.092 \mathrm{ab}$ & $66.667 \pm 4.910 \mathrm{a}$ \\
\hline & & male & $0.787 \pm 0.01 \mathrm{ab}$ & $0.596 \pm 0.015$ bcde & $0.896 \pm 0.007$ abcde & $0.446 \pm 0.046 \mathrm{bcd}$ & $59.000 \pm 1.528$ bcde \\
\hline & \multirow[t]{2}{*}{$\mathrm{Ca}$} & female & $0.788 \pm 0.001 \mathrm{a}$ & $0.538 \pm 0.011$ efg & $0.861 \pm 0.001 \mathrm{efg}$ & $0.573 \pm 0.021 \mathrm{ab}$ & $53.000 \pm 1.000$ ef \\
\hline & & male & $0.795 \pm 0.005 \mathrm{a}$ & $0.583 \pm 0.022 \mathrm{cdef}$ & $0.887 \pm 0.011 \mathrm{bcdef}$ & $0.482 \pm 0.056 \mathrm{bc}$ & $58.000 \pm 2.082 \mathrm{cde}$ \\
\hline & \multirow[t]{15}{*}{$\mathrm{NaCl}+\mathrm{Ca}$} & female & $0.794 \pm 0.017 \mathrm{a}$ & $0.499 \pm 0.021 \mathrm{~g}$ & $0.859 \pm 0.004 \mathrm{fg}$ & $0.528 \pm 0.03 \mathrm{ab}$ & $56.333 \pm 0.882 \mathrm{de}$ \\
\hline & & male & $0.801 \pm 0.006 \mathrm{a}$ & $0.571 \pm 0.01 \mathrm{def}$ & $0.878 \pm 0.009 \mathrm{cdefg}$ & $0.648 \pm 0.041 \mathrm{a}$ & $49.667 \pm 2.028 \mathrm{f}$ \\
\hline & & $P>\mathrm{F}_{\mathrm{S}}$ & $0.188 \mathrm{~ns}$ & $0.417 \mathrm{~ns}$ & $0.109 \mathrm{~ns}$ & $0.946 \mathrm{~ns}$ & $0.073 \mathrm{~ns}$ \\
\hline & & $P>\mathrm{F}_{\mathrm{W}}$ & $0.019^{*}$ & $0.000 * * *$ & $0.001 * * *$ & $0.000 * * *$ & $0.005^{* *}$ \\
\hline & & $P>\mathrm{F}_{\mathrm{Na}}$ & $0.138 \mathrm{~ns}$ & $0.000 * * *$ & $0.000 * * *$ & $0.000 * * *$ & $0.003 * *$ \\
\hline & & $P>\mathrm{F}_{\mathrm{Ca}}$ & $0.005^{* *}$ & $0.261 \mathrm{~ns}$ & $0.252 \mathrm{~ns}$ & $0.001 * * *$ & $0.001 * * *$ \\
\hline & & $P>\mathrm{F}_{\mathrm{Na} \times \mathrm{Ca}}$ & $0.694 \mathrm{~ns}$ & $0.000 * * *$ & $0.003 * *$ & $0.000 * * *$ & $0.018^{*}$ \\
\hline & & $P>\mathrm{F}_{\mathrm{S} \times \mathrm{W}}$ & $0.556 \mathrm{~ns}$ & $0.261 \mathrm{~ns}$ & $0.284 \mathrm{~ns}$ & $0.313 \mathrm{~ns}$ & $0.845 \mathrm{~ns}$ \\
\hline & & $P>\mathrm{F}_{\mathrm{S} \times \mathrm{Na}}$ & $0.631 \mathrm{~ns}$ & $0.025^{*}$ & $0.098 \mathrm{~ns}$ & $0.048^{*}$ & $0.003 * *$ \\
\hline & & $P>\mathrm{F}_{\mathrm{S} \times \mathrm{Ca}}$ & $0.694 \mathrm{~ns}$ & $0.536 \mathrm{~ns}$ & $0.671 \mathrm{~ns}$ & $0.801 \mathrm{~ns}$ & $0.027 *$ \\
\hline & & $P>\mathrm{F}_{\mathrm{S} \times \mathrm{Na} \times \mathrm{Ca}}$ & $0.678 \mathrm{~ns}$ & $0.223 \mathrm{~ns}$ & $0.518 \mathrm{~ns}$ & $0.708 \mathrm{~ns}$ & $0.907 \mathrm{~ns}$ \\
\hline & & $P>\mathrm{F}_{\mathrm{W} \times \mathrm{Na}}$ & $0.499 \mathrm{~ns}$ & $0.055 \mathrm{~ns}$ & $0.277 \mathrm{~ns}$ & $0.076 \mathrm{~ns}$ & $0.372 \mathrm{~ns}$ \\
\hline & & $P>\mathrm{F}_{\mathrm{W} \times \mathrm{Ca}}$ & $0.616 \mathrm{~ns}$ & $0.000 * * *$ & $0.001 * * *$ & $0.000 * * *$ & $0.000 * * *$ \\
\hline & & $P>\mathrm{F}_{\mathrm{W} \times \mathrm{Na} \times \mathrm{Ca}}$ & $0.861 \mathrm{~ns}$ & $0.838 \mathrm{~ns}$ & $0.419 \mathrm{~ns}$ & $0.918 \mathrm{~ns}$ & $0.018^{*}$ \\
\hline & & $\begin{array}{l}P> \\
\mathrm{F}_{\mathrm{S} \times \mathrm{W} \times \mathrm{Na} \times \mathrm{Ca}}\end{array}$ & $0.677 \mathrm{~ns}$ & $0.000^{* * *}$ & $0.005^{* *}$ & $0.013^{*}$ & $0.273 \mathrm{~ns}$ \\
\hline
\end{tabular}

of $\mathrm{Na}: \mathrm{Ca}$ in female and male clones. Both treatments increased the values of $\mathrm{Na}$ :Ca to more than 20 -fold in both clones compared with their individual controls under well-watered conditions. However, $\mathrm{Ca}$ treatment under waterlogging caused no effect on the ratio of $\mathrm{Na}: \mathrm{Ca}$ of both clones compared with their individual controls. The female clones had significantly higher $\mathrm{Na}$ :Ca ratio than male clones after salinity and combined salinity and $\mathrm{Ca}$ treatments under waterlogging. The effects of sex, waterlogging, salt, $\mathrm{Ca}$ and their interaction are also shown in Table 1.

Under well-watered conditions, the salinity, $\mathrm{Ca}$, and combined salinity and $\mathrm{Ca}$ treatments decreased $\mathrm{P}_{\mathrm{N}}, \mathrm{g}_{\mathrm{s}}$, and WUEi. The treatments significantly increased $\mathrm{E}$ but caused no effect on $\mathrm{c}_{\mathrm{i}}$ (Table 2) in female and male clones compared with their controls. No significant differences in $\mathrm{P}_{\mathrm{N}}, \mathrm{g}_{\mathrm{s}}, \mathrm{WUEi}, \mathrm{E}$, and $\mathrm{c}_{\mathrm{i}}$ were found among male or female clones in different treatments. In addition, the male clones had significantly higher $\mathrm{P}_{\mathrm{N}}$ than the female clones in salinity treatment, whereas no significant differences in $\mathrm{P}_{\mathrm{N}}, \mathrm{g}_{\mathrm{s}}, \mathrm{WUEi}, \mathrm{E}$, and $\mathrm{c}_{\mathrm{i}}$ were identified between both sexes in other treatments.

Waterlogging decreased $\mathrm{P}_{\mathrm{N}}, \mathrm{g}_{\mathrm{s}}$, WUEi, E, and $\mathrm{c}_{\mathrm{i}}$ in both clones compared with the controls. The salinity, $\mathrm{Ca}$, and combined salinity and $\mathrm{Ca}$ treatments under waterlogging decreased $\mathrm{P}_{\mathrm{N}}, \mathrm{g}_{\mathrm{s}}$, and $\mathrm{E}$, increased WUEi, and showed no effect on $\mathrm{c}_{\mathrm{i}}$ in both clones compared with their controls under well-watered conditions. Under waterlogging, significant variations in $\mathrm{P}_{\mathrm{N}}$ (combined salinity and $\mathrm{Ca}$ treatments in male clones), $\mathrm{g}_{\mathrm{s}}$ (salinity and combined salinity and $\mathrm{Ca}$ treatments in female clones), and WUEi (salinity and combined salinity and $\mathrm{Ca}$ treatments in female clones) were observed compared with their individual controls under well-watered conditions. In addition, the male clones had significantly higher $\mathrm{c}_{\mathrm{i}}$ content than the female clones after the combined salinity and $\mathrm{Ca}$ treatment under 


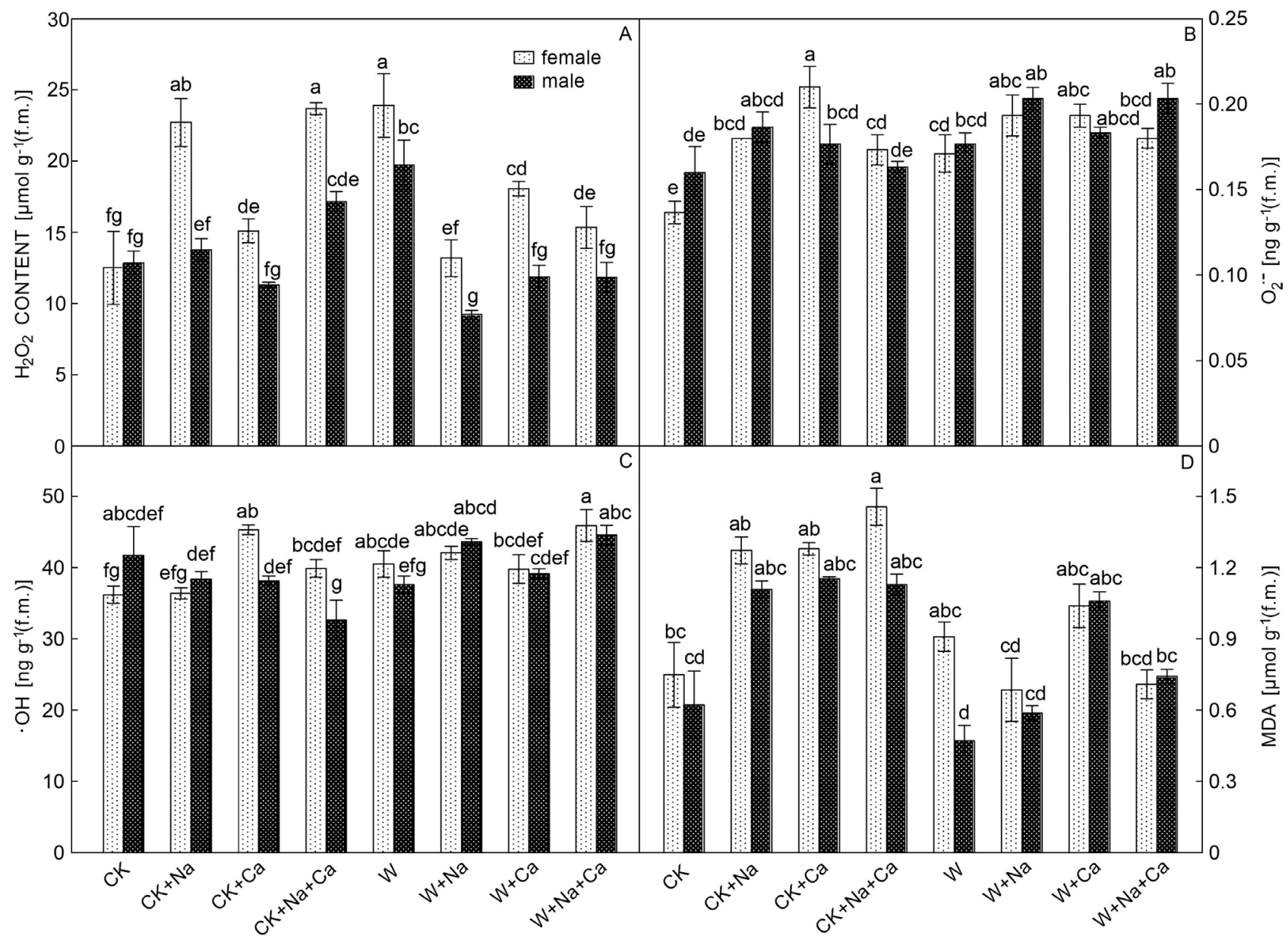

Fig. 1. Hydrogen peroxide $\left(\mathrm{H}_{2} \mathrm{O}_{2}, A\right)$, superoxide radical $\left(\mathrm{O}_{2}{ }^{--}, B\right)$, hydroxyl radical ( $\left.\mathrm{OH}, C\right)$, and malondialdehyde (MDA, $\left.D\right)$ content in male and female Populus deltoides. $\mathrm{CK}$ - control treatment, $\mathrm{CK}+\mathrm{Na}$ - salinity treatment, $\mathrm{CK}+\mathrm{Ca}-$ calcium treatment, $\mathrm{CK}+\mathrm{Na}+\mathrm{Ca}-$ combined salinity and calcium treatment, $\mathrm{W}$ - waterlogging treatment, $\mathrm{W}+\mathrm{Na}$ - salinity treatment under waterlogging, $\mathrm{W}+\mathrm{Ca}-$ calcium treatment under waterlogging, $\mathrm{W}+\mathrm{Na}+\mathrm{Ca}$ - combined salinity and calcium treatment under waterlogging. Means $\pm \mathrm{SEs}, n=6$; different letters above the columns indicate significant differences at $P<0.05$ according to Tukey's test.

waterlogging, whereas no significant differences in $\mathrm{P}_{\mathrm{N}}, \mathrm{g}_{\mathrm{s}}$, WUEi, E, and $c_{i}$ were recognized between both sexes after other treatments under waterlogging. The effects of sex, waterlogging, salt, $\mathrm{Ca}$ and their interaction effects were also shown in Table 2 .

Under well-watered conditions, salinity, $\mathrm{Ca}$, and combined salinity and $\mathrm{Ca}$ treatments showed no significant effect on $\mathrm{F}_{\mathrm{v}} / \mathrm{F}_{\mathrm{m}}$, Yield, $\mathrm{qP}, \mathrm{qN}$, and ETR (Table 3) in female clones compared with the controls, whereas salinity treatment significantly decreased Yield, $\mathrm{qP}$, and ETR and significantly increased $\mathrm{qN}$ in male clones. $\mathrm{F}_{\mathrm{v}} / \mathrm{F}_{\mathrm{m}}$, Yield, $\mathrm{qP}$, $\mathrm{qN}$, and ETR exhibited no significant difference among female clones under different treatments. The Yield, $\mathrm{qP}$, and ETR of male clones in salinity treatment were significantly lower than those of their counterparts in $\mathrm{Ca}$ and combined salinity and $\mathrm{Ca}$ treatments, whereas the $\mathrm{qN}$ was significantly higher in salinity treatment than in the other two treatments. In addition, significant differences in Yield, qP, qN, and ETR between both clones were found only in salinity treatment. No significant differences in $\mathrm{F}_{\mathrm{v}} / \mathrm{F}_{\mathrm{m}}$, Yield, $\mathrm{qP}, \mathrm{qN}$, and ETR between both sexes were observed under other treatments.

Waterlogging showed no significant effect on $\mathrm{F}_{\mathrm{v}} / \mathrm{F}_{\mathrm{m}}$, Yield, $\mathrm{qP}, \mathrm{qN}$, and ETR in female and male clones compared with the controls. The salinity, $\mathrm{Ca}$, and combined salinity and $\mathrm{Ca}$ treatments under waterlogging increased qN, decreased Yield, qP, and ETR; and showed minimal effect on $F_{v} / F_{m}$ in both clones compared with their individual controls under well-watered conditions. However, significant variations were observed in Yield (in both clones under $\mathrm{Ca}$ and salinity and $\mathrm{Ca}$ treatments), $\mathrm{qP}$ (in female clones all three treatments), $\mathrm{qN}$ (in both clones under $\mathrm{Ca}$ and combined salinity and $\mathrm{Ca}$ treatments), and ETR (in both clones under combined salinity and $\mathrm{Ca}$ treatment) under waterlogging. Slight differences in $\mathrm{F}_{\mathrm{v}} / \mathrm{F}_{\mathrm{m}}$, Yield, $\mathrm{qP}, \mathrm{qN}$, and ETR were found among male or female clones under the three treatments in waterlogging. In addition, the male clones had significantly higher Yield levels than the female clones after $\mathrm{Ca}$ and combined salinity and $\mathrm{Ca}$ treatments under waterlogging stress conditions and significantly higher $\mathrm{qP}$ levels than the females in $\mathrm{Ca}$ treatment. The effects of sex, waterlogging, salt, $\mathrm{Ca}$ and 


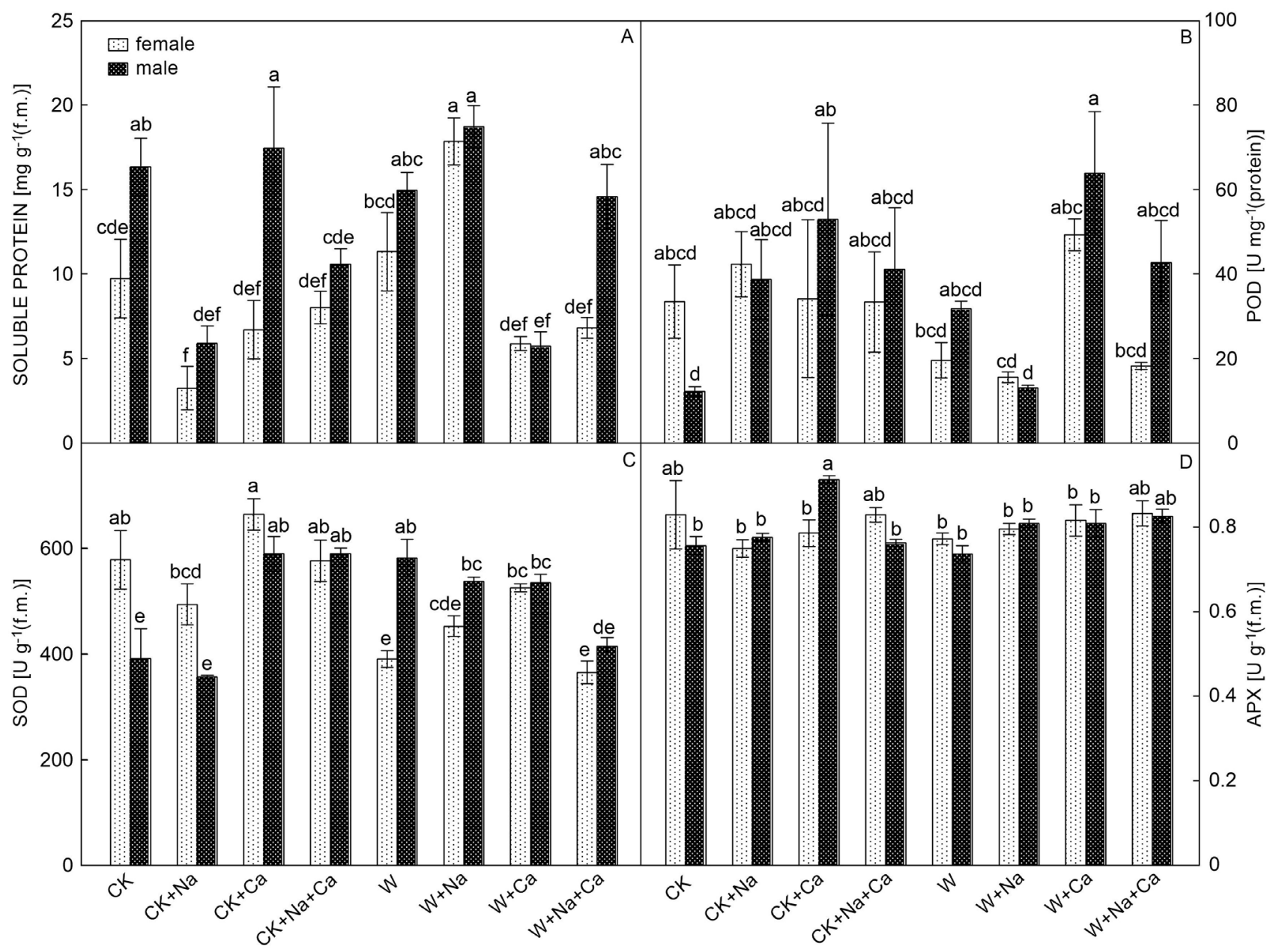

Fig. 2. Soluble protein content $(A)$ and activities of peroxidase (POD, $B$ ), superoxide dismutase (SOD, $C$ ), and ascorbate peroxidase (APX, $D$ ) in male and female Populus deltoids. Means \pm SEs, $n=6$; different letters above the columns indicate significantly differences at $P<0.05$ according to Tukey's test. For explanation of abbreviations - see Fig. 1.

their interaction effects were also shown in Table 3.

Under well-watered conditions, the salinity, Ca, and combined salinity and $\mathrm{Ca}$ treatments increased the contents of $\mathrm{H}_{2} \mathrm{O}_{2}$ (Fig. 1A), $\mathrm{O}_{2}{ }^{-}$(Fig. 1B), $\cdot$ OH (Fig. 1C), and MDA (Fig. $1 D$ ) in female and male clones compared with their controls. The female clones had higher levels of $\mathrm{H}_{2} \mathrm{O}_{2}, \mathrm{O}_{2} \cdot-\mathrm{OH}$, and MDA than the male clones. In female clones, salinity treatment could significantly increase the levels of $\mathrm{H}_{2} \mathrm{O}_{2}$ and $\mathrm{O}_{2}^{-}$; $\mathrm{Ca}$ treatment could significantly increase the levels of $\mathrm{H}_{2} \mathrm{O}_{2}, \mathrm{O}_{2}{ }^{--}$, and $\cdot \mathrm{OH}$; the combined salinity and $\mathrm{Ca}$ treatment could significantly increase the levels of $\mathrm{H}_{2} \mathrm{O}_{2}, \mathrm{O}_{2}{ }^{-}$, and MDA. However, only the combined salinity and $\mathrm{Ca}$ treatment increased the level of $\mathrm{H}_{2} \mathrm{O}_{2}$ in male clones. Slight differences in the levels of $\mathrm{H}_{2} \mathrm{O}_{2}, \mathrm{O}_{2}^{--}, \cdot \mathrm{OH}$, and MDA were detected in both clones under the three treatments. In addition, the female clones had significantly higher levels of $\mathrm{H}_{2} \mathrm{O}_{2}$ than the male clones in all three treatments, significantly higher levels of $\mathrm{O}_{2}^{-}$in $\mathrm{Ca}$ treatment, and significantly higher levels of $\cdot \mathrm{OH}$ in $\mathrm{Ca}$ and combined salinity and $\mathrm{Ca}$ treatments than the male clones.

Waterlogging significantly increased the content of $\mathrm{H}_{2} \mathrm{O}_{2}, \mathrm{O}_{2}{ }^{--}$, and $\mathrm{OH}$ in female clones but did not significantly affect the content of $\mathrm{O}_{2}{ }^{--}$and $\mathrm{OH}$ in male clones. All three treatments under waterlogging conditions had limited effects on the content of $\mathrm{H}_{2} \mathrm{O}_{2}$, $\mathrm{O}_{2}{ }^{--}, \mathrm{OH}$, and MDA in both clones compared with their individual controls under well-watered conditions. Under waterlogging, significant variations were observed in $\mathrm{H}_{2} \mathrm{O}_{2}$ (in both clones under salinity and combined salinity and $\mathrm{Ca}$ treatments), $\mathrm{O}_{2}{ }^{-}$(in male clones under combined salinity and $\mathrm{Ca}$ treatment), $\mathrm{OH}$ (in both clones under combined salinity and Ca treatment), and MDA (in female clones under salinity and combined salinity and $\mathrm{Ca}$ treatments) compared with their individual controls under wellwatered conditions. Slight differences were noted in their content in all treatments under waterlogging. In addition, the female clones had significantly higher content of $\mathrm{H}_{2} \mathrm{O}_{2}$ than the male clones in all three treatments under waterlogging stress conditions. However, no significant differences between both sexes were identified in all treatments. The effects of sex, waterlogging, salt, $\mathrm{Ca}$, and their interaction effects could be found in Table 1 Suppl.

Under well-watered conditions, the soluble protein 
content (Fig. 2A) significantly decreased after salinity treatment in both clones and after combined salinity and $\mathrm{Ca}$ treatment in male clones. No significant variations in the POD (Fig. 2B), SOD (Fig. 2C), and APX (Fig. 2D) activities were found when the female clones were exposed to all treatments. However, in male clones, significant increases in the POD and APX activities were observed after $\mathrm{Ca}$ treatment, whereas significant increases in SOD activities were induced during $\mathrm{Ca}$ and combined salinity and $\mathrm{Ca}$ treatments. Slight differences were noted in the soluble protein content and POD, SOD, and APX activities of female clones under the three treatments, whereas for the male clones, $\mathrm{Ca}$ treatment could induce significantly higher content of soluble proteins and APX activity than the other two treatments. In addition, the male clones had significantly higher soluble protein content and APX activity in Ca treatment than the female clones.

Waterlogging caused no significant effect on soluble protein contents and POD and APX activities in either female nor male clones. However, the SOD activities in male clones significantly increased under waterlogging stress but significantly decreased in female clones. Under waterlogging stress conditions, the soluble protein contents were significantly increased by salinity treatment of both clones, whereas the SOD activities in male clones were significantly increased by salinity treatment, significantly decreased by $\mathrm{Ca}$ treatment in female clones, and significantly decreased by the combined salinity and $\mathrm{Ca}$ treatment in both clones. Under waterlogging, minimal differences in SOD and APX activities were observed in both clones under all treatments. In addition, the male clones had significantly higher soluble protein content and SOD activity than the female clones after the combined salinity and $\mathrm{Ca}$ treatment under waterlogging. The effects of sex, waterlogging, salt, $\mathrm{Ca}$ and their interaction effects could be found in Table 2 Suppl.

\section{Discussion}

Previous studies have suggested that specific $P$. deltoides clones and hybrids could survive well under $200 \mathrm{mM} \mathrm{NaCl}$ treatment (Bray et al. 1991, Fung et al. 1998, Singh et al. 2000). Specifically, $10 \mathrm{mM} \mathrm{Ca}$ is necessary for alleviating stress-induced damages in many plants (Cramer 2002, Cabañero et al. 2004, Murillo-Amador et al. 2006, Parvin 2016, Yang et al. 2016). A total of $20 \mathrm{mM} \mathrm{Ca}$ was used to enhance the germination, growth, and yield of plants under $\mathrm{NaCl}$ stress (Lopez and Satti 1996, Salahshoor and Kazemi 2016). However, leaf chlorosis, leaf necrosis, and leaf abscission, after $15-\mathrm{d} 100 \mathrm{mM} \mathrm{NaCl}$ treatment or $10 \mathrm{mM}$ $\mathrm{Ca}$ treatment suggested that female and male $P$. deltoides clones are sensitive to $\mathrm{Na}$ and $\mathrm{Ca}$ under well-watered or waterlogging conditions. Therefore, although $P$. deltoides is a desirable tree species for riparian-protective forests due to its strong waterlogging tolerance (Regehr et al. 1975, Yang et al. 2011, Miao et al. 2017), it is unsuitable for the construction of protection forests in estuary and intertidal zones with high concentrations of $\mathrm{Na}$ and $\mathrm{Ca}$.

The combination of salinity and waterlogging stresses has a more significant influence on the growth, anatomical traits, antioxidative traits, and elemental toxicity of nonhalophytes than one type of stress (Alhdad et al. 2013, Zeng et al. 2013, Haddadi et al. 2016). Na-Ca interactions via exogenous $\mathrm{Ca}$ application could directly regulate plant growth, photosynthesis, osmotic stress, mineral nutrition, and water and ion transport, thereby mitigating the adverse effects of salt-induced ionic toxicity (Rengel 1992, Lopez and Satti 1996, Singh et al. 1999, Cramer 2002, Girija et al. 2002, Cabañero and Carvajal 2004, Murillo-Amador et al. 2006, Tuna et al. 2007, Jian et al. 2009, Tattini and Traversi 2009, Salahshoor and Kazemi 2016, Srivastava et al. 2013, An et al. 2014). However, research rarely focused on the plant response to combined salinity and Ca stress under well-watered or waterlogging conditions. Salinity and $\mathrm{Ca}$ under well-watered conditions could increase the $\mathrm{K}$ and $\mathrm{Ca}$ content, ROS production, and antioxidant enzyme activities and decrease $\mathrm{P}_{\mathrm{N}}, \mathrm{g}_{\mathrm{s}}, \mathrm{WUEi}$, Yield, and ETR. Further analyses of these physiological and biochemical traits suggested that salinity and $\mathrm{Ca}$ cause similar responses, and their combination is not more serious than individual effects. In addition, an independent process occurs under the combined effects of salinity and $\mathrm{Ca}$. The lack of sharp variation in $\mathrm{Na}$ and $\mathrm{Ca}$ content in leaves suggests that the ionic toxicity from salinity and Ca possibly affected the root systems but not the aerial parts under well-watered conditions. Waterlogging could notably stimulate $\mathrm{Na}$ uptake in leaves under abundant $\mathrm{NaCl}$. However, this condition had no evident effects on $\mathrm{Ca}$ uptake under abundant $\mathrm{Ca}$. The decreased $\mathrm{Na}$ content and increased $\mathrm{K}$ and $\mathrm{Ca}$ content of male clones suggest that male clones can maintain ion homeostasis better than female clones under salinity, $\mathrm{Ca}$, and combined salinity and $\mathrm{Ca}$ treatments (Cramer 2002).

The sex-specific morphological, physiological, biochemical, ultrastructural, transcriptional, and proteomic responses to waterlogging (Letts et al. 2008, Nielsen et al. 2010, Rood et al. 2010, Yang et al. 2011, Miao et al. 2017) and salinity (Chen et al. 2010, Chen et al. 2011, Jiang et al. 2012, Xu et al. 2015, Li et al. 2016) stresses in poplars have been well studied. The majority of studies concluded that females are more sensitive to salinity and usually experience greater negative effects than males. However, Robinson et al. (2014) suggested that no evidence of sexual dimorphism or differential resource investment strategies existed between males and females in mature leaves of mature $P$. tremula using whole-genome oligonucleotide microarrays and RNAsequencing. McKown et al. (2017) also failed to detect sexual dimorphism in more than 1300 individuals from $P$. trichocarpa and $P$. balsamifera. Our previous studies have also demonstrated that male $P$. deltoides clones develop better cellular defense mechanisms against waterlogging/flooding stress than their female counterpart, thereby making them less susceptible (Yang et al. 2011, Miao et al. 2017). In the present study, differences in certain parameters were insignificant between the two sexes under stressed conditions. However, sex-specific differences in $\mathrm{P}_{\mathrm{N}}, \mathrm{c}_{\mathrm{i}}$, Yield, qP, qN, ETR, $\mathrm{O}_{2}{ }^{--}, \mathrm{OH}, \mathrm{H}_{2} \mathrm{O}_{2}$, soluble protein content, and SOD activity under certain 
stressed conditions suggest that female $P$. deltoides clones are more sensitive to salinity, $\mathrm{Ca}$, and combined salinity and $\mathrm{Ca}$ treatments than their male counterparts under well-watered or waterlogging conditions. The oxidative stress might limit the photosynthetic $\mathrm{CO}_{2}$ assimilation and damage to photosystems.

In conclusion, both sexes of $P$. deltoides are waterlogging tolerant but sensitive to salinity and Ca. Under well-watered conditions, salinity stress could especially increase the $\mathrm{Na}$ content of female clones, whereas salinity and Ca could especially increase the $\mathrm{K}$ and $\mathrm{Ca}$ content in male clones. Waterlogging could remarkably stimulate $\mathrm{Na}$ uptake in leaves under salinity, especially in female $P$. deltoides. However, waterlogging had no effects on $\mathrm{Ca}$ uptake under abundant $\mathrm{Ca}$. Salinity and $\mathrm{Ca}$ caused similar physiological and biochemical responses, and their combination had not more significant effect than single stresses, suggesting that independent processes occur under the combined effects of salinity and $\mathrm{Ca}$. The lower $\mathrm{Na}$ content and higher $\mathrm{K}$ and $\mathrm{Ca}$ content in male clones suggested that males have better abilities to maintain ion homeostasis than females. The sex-specific differences in $\mathrm{P}_{\mathrm{N}}, \mathrm{c}_{\mathrm{i}}$, Yield, qP, qN, ETR, $\mathrm{O}_{2}{ }^{--}, \mathrm{OH}, \mathrm{H}_{2} \mathrm{O}_{2}$, soluble protein content, and SOD activity under certain stresses suggest that $P$. deltoides female clones are more sensitive to salinity, $\mathrm{Ca}$, and combined salinity and $\mathrm{Ca}$ treatments than male clones either under well-watered or waterlogging conditions. This study sheds a new light on the possible construction of $P$. deltoides protected forests and increases the understanding of sexually dimorphic responses to combined multifactorial stresses.

\section{References}

Alhdad, G.M., Seal, C.E., Al-Azzawi, M.J., Flowers, T.J.: The effect of combined salinity and waterlogging on the halophyte Suaeda maritima: the role of antioxidants. - Environ. exp. Bot. 87: 120-125, 2013.

An, P., Li, X., Zheng, Y., Eneji, A.E., Inanaga, S.: Calcium effects on root cell wall composition and ion contents in two soybean cultivars under salinity stress. - Can. J. Plant Sci. 94: 733-740, 2014.

Bradford, M.M.: A rapid and sensitive method for the quantitation of microgram quantities of protein utilizing the principle of protein-dye binding. - Anal. Biochem. 72: 248-254, 1976.

Bray, L., Chriqui, D., Gloux, K., Rudulier, D.L., Meyer, M., Peduzzi, J.: Betaines and free amino acids in salt stressed vitroplants and winter resting buds of Populus trichocarpa $\times$ deltoides. - Physiol. Plant. 83: 136-143, 1991.

Cabañero, F.J., Carvajal, M.: Does calcium determine water uptake under saline conditions in pepper plants, or is it water flux which determines calcium uptake? - Plant Sci. 166: 443450, 2004

Cao, F.L., Conner, W.H.: Selection of flood-tolerant Populus deltoides clones for reforestation projects in China. - Forest Ecol. Manage. 117: 211-220, 1999.

Chen, F., Zhang, S., Jiang, H., Ma, W., Korpelainen, H., Li, C.: Comparative proteomics analysis of salt response reveals sex-related photosynthetic inhibition by salinity in Populus cathayana cuttings. - J. Proteome Res. 10: 3944-3958, 2011.

Chen, L., Zhang, S., Zhao, H., Korpelainen, H., Li, C.: Sex- related adaptive responses to interaction of drought and salinity in Populus yunnanensis. - Plant Cell Environ. 33: 1767-1778, 2010

Cooper, D.J., D’Amico, D.R., Scott, M.L.: Physiological and morphological response patterns of Populus deltoides to alluvial ground water pumping. - Environ. Manage. 31: 215226, 2003.

Cramer, G.R.: Sodium-calcium interactions under salinity stress. Salinity: Environment - Plants - Molecules. - Springer, Dordrecht 2002.

Fung, L.E., Wang, S.S., Altman, A., Hütterman, A.: Effect of $\mathrm{NaCl}$ on growth, photosynthesis, ion and water relations of four poplar genotypes. - Forest Ecol. Manage. 107: 135-146, 1998.

Gao, H., Jia, Y., Guo, S., Lv, G., Wang, T., Juan, L.: Exogenous calcium affects nitrogen metabolism in root-zone hypoxiastressed muskmelon roots and enhances short-term hypoxia tolerance. - J. Plant Physiol. 168: 1217-1225, 2011.

Gladwin, D.N., Roelle, J.E.: Survival of plains cottonwood (Populus deltoides subsp. monilifera) and saltcedar (Tamarix ramossima) seedlings in response to flooding. - Wetlands 18: 669-674, 1998.

Girija, C., Smith, B.N., Swamy, P.M.: Interactive effects of sodium chloride and calcium chloride on the accumulation of proline and glycine betaine in peanut (Arachis hypogaea L.). - Environ. exp. Bot. 47: 1-10, 2002.

Haddadi, B.S., Hassanpour, H., Niknam, V.: Effect of salinity and waterlogging on growth, anatomical and antioxidative responses in Mentha aquatica L. - Acta Physiol. Plant. 38: 1-11, 2016.

Han, C., Chan, Z., Yang, F.: Comparative analyses of universal extraction buffers for assay of stress related biochemical and physiological parameters. - Preparative Biochem. Biotechnol. 45: 684-695, 2015.

He, L., Lu, X., Tian, J., Yang, Y., Li, B., Li, J., Guo. S.: Proteomic analysis of the effects of exogenous calcium on hypoxicresponsive proteins in cucumber roots. - Proteome Sci. 10: 42, 2012.

Jian, S., Dai, S., Wang, R., Chen, S., Li, N., Zhou, X., Lu, C., Shen, X., Zheng, X., Hu, Z., Zhang, Z., Song J., Xu, Y.: Calcium mediates root $\mathrm{K}^{+} / \mathrm{Na}^{+}$homeostasis in poplar species differing in salt tolerance. - Tree Physiol. 29: 1175-1186, 2009.

Jiang, H., Peng, S., Sheng, Z., Li, X., Korpelainen, H., Li, C.: Transcriptional profiling analysis in Populus yunnanensis provides insights into molecular mechanisms of sexual differences in salinity tolerance. - J. exp. Bot. 63: 3709-3726, 2012.

Juvany, M., Munné-Bosch, S.: Sex-related differences in stress tolerance in dioecious plants: a critical appraisal in a physiological context. - J. exp. Bot. 66: 451-454, 2015.

Letts, M.G., Phelan, C.A., Johnson, D.R.E., Rood, S.B.: Seasonal photosynthetic gas exchange and leaf reflectance characteristics of male and female cottonwoods in a riparian woodland. - Tree Physiol. 28: 1037, 2008.

Li, Y., Duan, B., Chen, J., Korpelainen, H., Niinemets, Ü., Li, C.: Males exhibit competitive advantages over females of Populus deltoides under salinity stress. - Tree Physiol. 36: 1573-1584, 2016.

Lin, K.H., Chiou, Y.K., Hwang, S.Y., Chen, L.F.O., Lo, H.F.: Calcium chloride enhances the antioxidative system of sweet potato (Ipomoea batatas) under flooding stress. - Ann. Appl. Biol. 152: 157-168, 2008.

Lopez, M.V., Satti, S.M.E.: Calcium and potassium-enhanced growth and yield of tomato under sodium chloride stress. Plant Sci. 114: 19-27, 1996. 
McKown, A.D., Klápště, J., Guy, R.D., Soolanayakanahally, R.Y., La Mantia, J., Porth, I., Skyba, O., Unda, F., Douglas, C.J., El-Kassaby, Y.A., Hamelin, R.C., Mansfield, S.D., Cronk, Q.: Sexual homomorphism in dioecious trees: extensive tests fail to detect sexual dimorphism in Populus. - Sci. Rep. 7: 1831, 2017.

Miao, L.F., Yang, F., Han, C.Y., Pu, Y.J., Ding, Y., Zhang, L.J.: Sex-specific responses to winter flooding, spring waterlogging and post-flooding recovery in Populus deltoides. - Sci. Rep. 7: $2534,2017$.

Murillo-Amador, B., Jones, H.G., Kaya, C., Aguilar, R.L., García-Hernández, J.L., Troyo-Diéguez, E., Avila-Serrano, N.Y., Rueda-Puente, E.: Effects of foliar application of calcium nitrate on growth and physiological attributes of cowpea (Vigna unguiculata L. Walp.) grown under salt stress. - Environ. exp. Bot. 58: 188-196, 2006.

Nielsen, J.L., Rood, S.B., Pearce, D.W., Letts, M.G., Jiskoot, H., Ehleringer, J.: Streamside trees: responses of male, female and hybrid cottonwoods to flooding. - Tree Physiol. 30: 14791488, 2010.

Oh, M.W., Nanjo, Y., Komatsu, S.: Gel-free proteomic analysis of soybean root proteins affected by calcium under flooding stress. - Front. Plant Sci. 5: 559, 2014.

Parvin, K., Ahamed, K.U., Islam, M.M., Haque, N.: Modulation of ion uptake in tomato (Lycopersicon esculentum L.) plants with exogenous application of calcium under salt stress condition. - J. Plant Sci. 22: 40-49, 2016.

Regehr, D.L., Bazzaz, F.A., Boggess, W.R.: Photosynthesis, transpiration and leaf conductance in Populus deltoides in relation to flooding and drought. - Photosynthetica 9: 52-61, 1975.

Rengel, Z.: The role of calcium in salt toxicity. - Plant Cell Environ. 15: 625-632, 1992.

Robinson, K.M., Delhomme, N., Mähler, N., Schiffthaler, B., Onskog, J., Albrectsen, B.R., Ingvarsson, P.K., Hvidsten, T.R., Jansson, S., Street, N.R.: Populus tremula (European aspen) shows no evidence of sexual dimorphism. - BMC Plant Biol. 14: 276, 2014.

Rood, S.B., Nielsen, J.L., Shenton, L., Gill, K.M., Letts, M.G.: Effects of flooding on leaf development, transpiration, and photosynthesis in narrow leaf cottonwood, a willow-like poplar. - Photosynth. Res. 104: 31-39, 2010.

Rowland, D.L.: Diversity in physiological and morphological characteristics of four cottonwood (Populus deltoides var. wislizenii) populations in New Mexico: evidence for a genetic component of variation. - Can. J. Forest Res. 31: 845-853,
2001.

Salahshoor, F., Kazemi, F.: Effect of calcium on reducing salt stress in seed germination and early growth stage of Festuca ovina L. - Plant Soil Environ. 62: 460-467, 2016.

Singh, M., Jain, M., Pant, R.C.: Clonal variability in photosynthetic and growth characteristics of Populus deltoides under saline irrigation. - Photosynthetica 36: 605-609, 2000.

Srivastava, A.K., Rai, A.N., Patade, V.Y., Suprasanna, P.: Calcium signaling and its significance in alleviating salt stress in plants. - In: Ahmad, P., Azooz, M.M., Prasad, M.N.V. (ed.): Salt Stress in Plants: Signalling, Omics and Adaptations. Pp.197-218. - Springer, New York 2013.

Tattini, M., Traversi, M.L.: On the mechanism of salt tolerance in olive (Olea europaea L.) under low- or high-Ca ${ }^{2+}$ supply. Environ. exp. Bot. 65: 72-81, 2009.

Tuna, A.L., Kaya, C., Ashraf, M., Altunlu, H., Yokas, I., Yagmur, B.: The effects of calcium sulphate on growth, membrane stability and nutrient uptake of tomato plants grown under salt stress. - Environ. exp. Bot. 59: 173-178, 2007.

Williams, V., Twine, S.: Flame photometeric method for sodium, potassium, and calcium. - In: Paech, K., Tracey, M.V. (ed.) Modern Methods of Plant Analysis. Vol. 5. Pp. 535. - Springer, Berlin 1960.

Xu, X., Li, Y., Wang, B., Hu, J., Liao, Y.: Salt stress induced sex-related spatial heterogeneity of gas exchange rates over the leaf surface in Populus cathayana, Rehd.. - Acta Physiol. Plant. 37: 1709, 2015.

Yang, B.Z., Liu, Z.B., Zhou, S.D., Ou, L.J., Dai, X.Z., Ma, Y.Q., Zhang, Z.Q., Chen, W.C., Li, X.F., Liang, C.L., Yang, S., Zou, X.X.: Exogenous $\mathrm{Ca}^{2+}$ alleviates waterlogging caused damages to pepper. - Photosynthetica 54: 1-11, 2016.

Yang, F., Han, C., Li, Z., Guo, Y., Chan, Z.: Dissecting tissueand species-specific responses of two Plantago species to waterlogging stress at physiological level. - Environ. exp. Bot. 109: 177-185, 2015.

Yang, F., Wang, Y., Wang, J., Deng, W., Liao, L., Li, M.: Different eco-physiological responses between male and female Populus deltoides clones to waterlogging stress. - Forest Ecol. Manage. 262: 1963-1971, 2011.

Yang, F., Wang, Y., Chan, Z.: Perspectives on screening winterflood-tolerant woody species in the riparian protection forests of the Three Gorges Reservoir. - PLoS ONE 9: e108725, 2014.

Zeng, F., Shabala, L., Zhou, M., Zhang, G., Shabala, S.: Barley responses to combined waterlogging and salinity stress: separating effects of oxygen deprivation and elemental toxicity. - Front. Plant Sci. 4: 313, 2013. 